\title{
1974 compilation of data on the GSJ geochemical reference samples JG-1 granodiorite and JB-1 basalt
}

\author{
Atsushi Ando, Hajime Kurasawa, Teiko Ohmori and \\ EIZO TAKEDA
}

Geological Survey of Japan, Kawasaki 213, Japan

(Received December 6, 1974)

\begin{abstract}
Analytical data received by October, 1974 for rock analyses, 53 minor elements and strontium isotopic ratios of the GSJ JG-1 granodiorite and JB-1 basalt are tabulated: Averages for both major and minor elements are given.
\end{abstract}

The Geological Survey of Japan has issued two geochemical reference rock samples GSJ JG-1 granodiorite and JB-1 basalt for the determination of major, minor, isotopic compositions and geological ages. Locations, geological notes and processing of the samples have been reported (Ando, 1967; Kurasawa, 1968). Data of rock analyses, 47 minor elements, strontium isotopic ratios $\left({ }^{87} \mathrm{Sr} /{ }^{86} \mathrm{Sr}\right)$, oxygen isotopic compositions and geological ages (K-Ar) received for these rocks by August, 1971; have previously been compiled (ANDo et al., 1971). Best values for the GSJ reference samples have also been proposed (FLANAGAN, 1973). This is a second compilation of data received by October, 1974 for the GSJ geochemical reference rock samples JG-1 and JB-1.

JG-1 (Porphyritic biotite granodiorite) was collected from Sori, about $100 \mathrm{~km}$ north of Tokyo, Gunma Prefecture, Japan. The Sori-granodiorite is exposed as a small mass of $6 \times 12 \mathrm{~km}$ intruding into Paleozoic formation. An age of $85 \mathrm{~m} . \mathrm{y}$ was given by K-Ar method for this sample (ShiBATA, 1968).

JB-1 (Titanaugite-olivine basalt) was collected from Myokanji-Toge, about $7 \mathrm{~km}$ NNW of Sasebo City, Kyushu, Japan. The basalt is exposed as a lava flow which constitutes an upper member of Kita-matsuura plateau basalt which is within the Japan Sea alkali rock province (Kurasawa, 1968). An age of $8 \mathrm{~m} . \mathrm{y}$ was given by the K-Ar method for another sample of the lava (Ozima et al., 1968). The age for JB-1 itself, however, has not been measured.

Additional data (received between August, 1971 and October, 1974) are tabulated

Publication authorized by the Director, Geological Survey of Japan. 
for rock analyses (Tables 1, 2, 3) along with grand averages and precisions for all data (received between June, 1967 and October, 1974) (Table 4). All reported values are presented for 53 minor elements (Table 5) and strontium isotopic ratios $\left({ }^{87} \mathrm{Sr} /{ }^{86} \mathrm{Sr}\right.$ ) (Table 6), since only a limited number of analytical results were reported in the previous compilation.

In the tables (av.): arithmetic mean and (avc.): arithmetic mean of the values within $2 \sigma$ are presented, because the results for many minor constituents are insufficient for a statistical evaluation.

We are indebted to all analysts who contributed data for these samples.

Any geochemists, geologists or anlytical chemists interested in participating in our program are invited to write to Liaison Officer of Standard Samples, Geochemical Research Section, Geological Survey of Japan, 135 Hisamoto, Takatsu-ku, Kawasaki, 213 Japan.

\section{ACKNOWLEGMENTS}

We thank Dr. D. S. MCKAY of NASA Manned Spacecraft Center, Houston, Texas, for his kind advice and for reading the manuscript.

\section{AA: Atomic absorption} spectrometry

(av.): Average value (arithmetic mean)

(avc.): Consensus average value (arithmetic mean of the values within $2 \sigma$ )

$\alpha$ or cntg.: $\alpha$ or $\gamma$-ray counting Chem.: Chemical method Chromatg.: Chromatography Color.: Colorimetry Comb.: Combustion method EPMA: Electron-probe microanalyser

\section{Abbreviations in the tables}

Fl. Phot.: Flame photometry

Fluo.: Fluorescence spectrophotometry

FT: Fission track method

Grav.: Gravimetry

ID.: Isotope dilution mass spectrometry

Kinetic: Catalytic reaction kinetics

M: Mass spectrometry

(n): Number of determinations

NAA: Neutron activation analysis
OS: Optical emission spectrometry

OS(DR): Direct reading emission spectrometry

PAA: Photon activation analysis

Photom.: Absorption spectrophotometry

Pyrohyd.: Pyrohydrolysis

Rapid: Rapid method

Rn: Radon method

XRF: X-ray fluorescence spectrometry

Vol.: Volumetry

$\sigma:$ Standard deviation 
Table 1. Rock analyses, JG-1 (\%)

\begin{tabular}{|c|c|c|c|c|c|c|c|c|}
\hline & 25 & 26 & 27 & 28 & 29 & 30 & 31 & 32 \\
\hline $\mathrm{SiO}_{2}$ & 7178 & 72.19 & 72.43 & 72.33 & 71.5 & 72.40 & 72.70 & 72.20 \\
\hline $\mathrm{TiO}_{2}$ & 0.26 & 0.26 & 0.26 & 0.28 & 0.09 & 0.28 & 0.29 & 0.27 \\
\hline $\mathrm{Al}_{2} \mathrm{O}_{3}$ & 14.10 & 14.21 & 14.07 & 14.23 & 14.6 & 14.49 & 14.08 & 14.30 \\
\hline $\mathrm{Fe}_{2} \mathrm{O}_{3}$ & 0.31 & 0.40 & 0.49 & 0.39 & 0.60 & - & - & 0.50 \\
\hline $\mathrm{FeO}$ & 1.63 & 1.62 & 1.50 & 1.60 & 1.42 & - & - & 1.67 \\
\hline $\mathrm{MnO}$ & 0.07 & 0.06 & 0.06 & 0.06 & 0.07 & 0.05 & 0.06 & 0.05 \\
\hline $\mathrm{MgO}$ & 0.79 & 0.76 & 0.79 & 0.78 & 0.71 & 0.95 & 0.74 & 0.80 \\
\hline $\mathrm{CaO}$ & 2.16 & 2.20 & 2.10 & 2.25 & 2.18 & 2.26 & 2.46 & 2.16 \\
\hline $\mathrm{Na}_{2} \mathrm{O}$ & 3.36 & 3.37 & 3.34 & 3.35 & 3.40 & 3.54 & 3.33 & 3.48 \\
\hline $\mathrm{K}_{2} \mathrm{O}$ & 3.96 & 3.91 & 4.06 & 3.93 & 3.98 & 3.92 & 3.93 & 4.00 \\
\hline $\mathrm{P}_{2} \mathrm{O}_{5}$ & 0.12 & 0.11 & 0.10 & 0.09 & - & - & 0.09 & 0.09 \\
\hline $\mathrm{H}_{2} \mathrm{O}+$ & & 0.48 & 0.61 & 0.50 & 0.31 & - & - & 0.67 \\
\hline $\mathrm{H}_{2} \mathrm{O}-$ & 0.83 & 0.08 & - & 0.11 & - & - & 0.08 & 0.04 \\
\hline $\mathrm{CO}_{2}$ & - & 0.07 & 0.08 & - & - & - & - & 0.11 \\
\hline $\mathrm{Cl}$ & - & - & - & - & - & - & - & - \\
\hline $\mathrm{F}$ & - & - & - & - & - & - & - & - \\
\hline Less $\mathrm{O}=\mathrm{F}+\mathrm{Cl}$ & - & - & - & - & - & - & - & - \\
\hline Sum & 99.37 & 99.72 & 99.89 & 99.90 & 98.86 & - & - & 100.34 \\
\hline $\begin{array}{l}\text { Total } \mathrm{Fe} \\
\text { as } \mathrm{Fe}_{2} \mathrm{O}_{3}\end{array}$ & 2.12 & 2.20 & 2.16 & 2.17 & 2.18 & 2.34 & 2.20 & 2.36 \\
\hline
\end{tabular}

25. BEASLEY (1972) XRF plus FeO (Chem. Vol.) Na \& K (Fl. Phot.); 26. OHTA (1972) conventional methods; 27. FRIESE (1972) ditto, 28. SHIRAHATA (1972) ditto; 29. RANDALL (1972) AA plus Ti \& FeO (Chem. Photom.); 30. Mori (1972) EPMA; 31. MARUYAMA and SUDA (1974) $\mathrm{SiO}_{2}$ (Grav. plus Color.) $\mathrm{Al}$, Ca \& $\mathrm{Mg}$ (EDTA Vol.) total $\mathrm{Fe}$ (Chem. Vol.) Mn(AA) Na \& K(Fl. Phot.) P(Chem. Photom.); 32. GAGNON (1974) $\mathrm{SiO}_{2}$ (Grav. plus $\mathrm{AA}$ ) $\mathrm{FeO}(\mathrm{Chem}$. Vol.) total $\mathrm{Fe}, \mathrm{Na}, \mathrm{K} \& \mathrm{Mn}(\mathrm{AA}) \mathrm{Ca} \& \mathrm{Mg}$ (EDTA Vol.).

Table 2. Rock analyses, JB-1 (\%)

\begin{tabular}{lrrrrrrrr}
\hline \hline & \multicolumn{1}{c}{18} & \multicolumn{1}{c}{19} & \multicolumn{1}{c}{20} & \multicolumn{1}{c}{21} & \multicolumn{1}{c}{22} & \multicolumn{1}{c}{23} & \multicolumn{1}{c}{24} & \multicolumn{1}{c}{5} \\
\hline $\mathrm{SiO}_{2}$ & 51.64 & 52.17 & 52.35 & 51.9 & 52.48 & 52.65 & 52.20 & 52.19 \\
$\mathrm{TiO}_{2}$ & 1.30 & 1.36 & 1.38 & 1.15 & 1.36 & 1.34 & 1.34 & 1.32 \\
$\mathrm{Al}_{2} \mathrm{O}_{3}$ & 14.38 & 14.54 & 14.44 & 14.7 & 14.34 & 14.57 & 14.50 & 14.54 \\
$\mathrm{Fe}_{2} \mathrm{O}_{3}$ & 2.24 & 2.21 & 2.40 & 3.06 & - & - & 2.42 & 2.47 \\
$\mathrm{FeO}$ & 5.98 & 6.00 & 5.91 & 5.42 & - & - & 6.00 & 5.98 \\
$\mathrm{MnO}$ & 0.16 & .0 .16 & 0.15 & 0.16 & - & 0.15 & 0.12 & 0.16 \\
$\mathrm{MgO}$ & 7.72 & 7.69 & 7.64 & 7.82 & 7.79 & 7.99 & 7.87 & 7.64 \\
$\mathrm{CaO}$ & 9.24 & 9.35 & 9.31 & 9.97 & 9.27 & 9.33 & 9.34 & 9.17 \\
$\mathrm{Na}_{2} \mathrm{O}$ & 2.84 & 2.72 & 2.77 & 2.81 & - & 2.85 & 2.90 & - \\
$\mathrm{K}_{2} \mathrm{O}$ & 1.40 & 1.37 & 1.40 & 1.50 & - & 1.41 & 1.50 & - \\
$\mathrm{P}_{2} \mathrm{O}_{5}$ & 0.29 & 0.27 & 0.26 & - & - & 0.26 & 0.25 & 0.25 \\
& & & & & & & & (Continued)
\end{tabular}




\begin{tabular}{|c|c|c|c|c|c|c|c|c|}
\hline $\mathrm{H}_{2} \mathrm{O}^{+}$ & & 0.94 & 0.90 & 0.93 & - & - & 0.75 & - \\
\hline $\mathrm{H}_{2} \mathrm{O}-$ & 2.02 & 0.84 & 0.85 & - & - & 0.99 & 0.85 & - \\
\hline $\mathrm{CO}_{2}$ & - & - & - & - & - & - & 0.21 & - \\
\hline $\mathrm{Cr}_{2} \mathrm{O}_{3}$ & - & - & 0.046 & - & - & - & - & - \\
\hline $\mathrm{Cl}$ & - & - & - & - & - & - & - & - \\
\hline $\mathrm{F}$ & - & - & - & - & - & - & - & - \\
\hline Sum & 99.21 & 99.82 & 99.81 & - & - & - & 100.25 & - \\
\hline $\begin{array}{l}\text { Total } \mathrm{Fe} \\
\text { as } \mathrm{Fe}_{2} \mathrm{O}_{3}\end{array}$ & 8.89 & 8.88 & 8.97 & 9.08 & 9.08 & 8.89 & 9.09 & 9.12 \\
\hline
\end{tabular}

18. BEASLEY (1972) XRF plus FeO(Chem. Vol.) Na \& K(Fl. Phot.); 19. OHTA (1972) conventional methods; 20. SHIRAHATA (1972) ditto; 21. RANDALL (1972)AA plus $\mathrm{Ti} \& \mathrm{FeO}$ (Chem. Photom.); 22. MORI (1972) EPMA; 23. MARUYAMA and SUDA (1974) $\mathrm{SiO}_{2}$ (Grav. plus Color.) $\mathrm{Al}, \mathrm{Ca} \& \mathrm{Mg}(\mathrm{EDTA}$ Vol.) total $\mathrm{Fe}(\mathrm{Chem}$. Vol.) $\mathrm{Mn}(\mathrm{AA})$ $\mathrm{Na} \& \mathrm{~K}$ (Fl. Phot.) P(Chem. Photom.); 24. GAGNON (1974) $\mathrm{SiO}_{2}$ (Grav. plus $\mathrm{AA}$ ) FeO (Chem. Vol.) total Fe, Na. K\& Mn(AA) Ca \& Mg(EDTA Vol.); 25. HAYATA (1974) $\mathrm{SiO}_{2}$ (Grav.) $\mathrm{Al}$, $\mathrm{Ca}, \mathrm{Mg}$, total $\mathrm{Fe}(\mathrm{EDTA}$ Vol.) $\mathrm{FeO}(\mathrm{Chem}$. Vol.) Mn(Color.) Ti \& $\mathrm{P}$ (Chem. Photom).

Table 3. Rock analyses, individual determinations (\%)

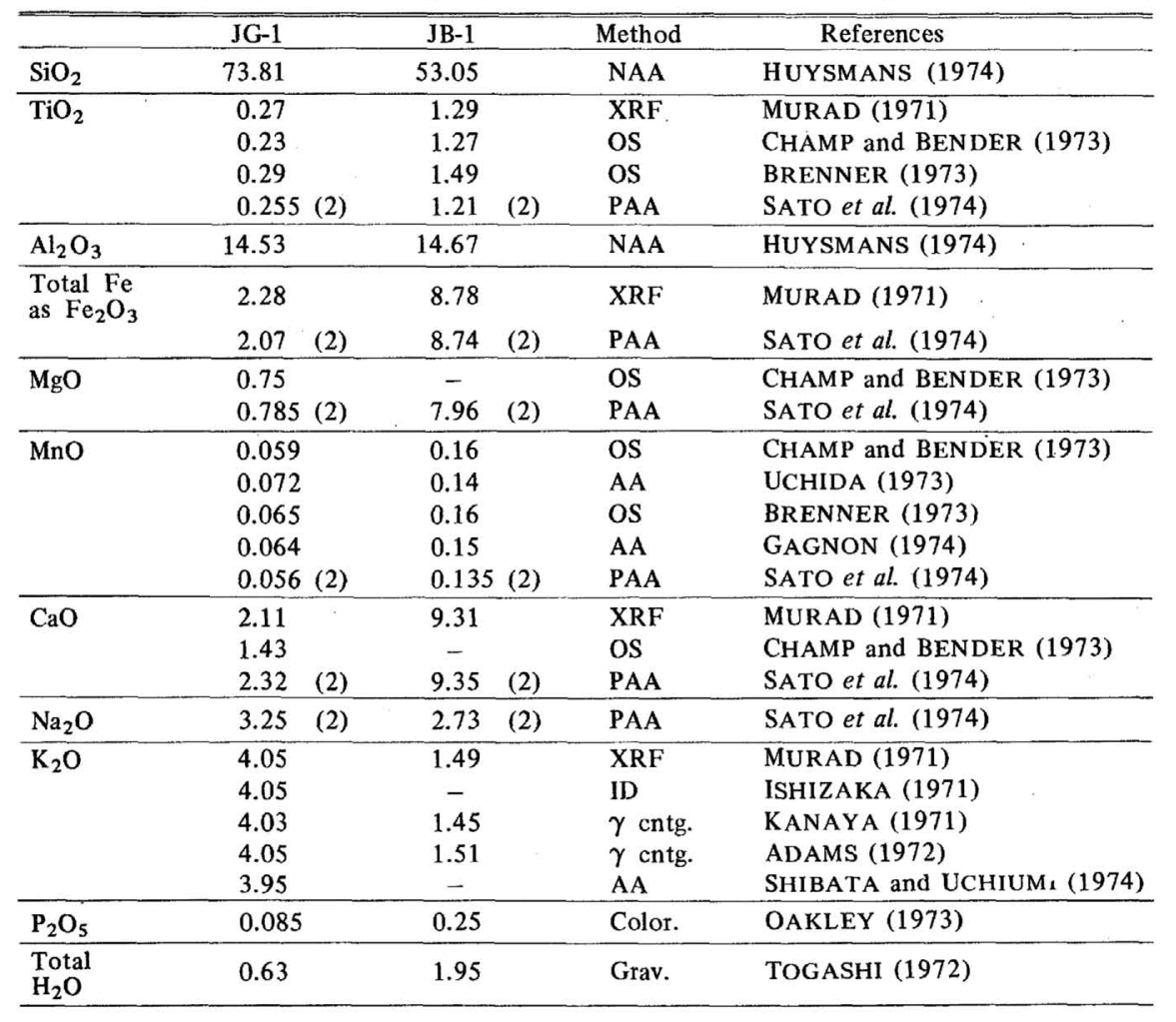


Table 4. Grand averages and precisions for JG-1 and JB-1 analyses

\begin{tabular}{|c|c|c|c|c|c|c|c|c|}
\hline & \multicolumn{4}{|c|}{ JG-1 } & \multicolumn{4}{|c|}{ JB-1 } \\
\hline & $\mathrm{n}$ & $\mathrm{X}$ & $\sigma$ & $\mathrm{Xc}$ & $\mathrm{n}$ & $\mathrm{Xc}$ & $\sigma$ & $\mathrm{Xc}$ \\
\hline $\mathrm{SiO}_{2}$ & 35 & 72.28 & 0.46 & 72.28 & 27 & 52.23 & 0.44 & 52.18 \\
\hline $\mathrm{TiO}_{2}$ & 40 & 0.26 & 0.042 & 0.27 & 31 & 1.35 & 0.10 & 1.34 \\
\hline $\mathrm{Al}_{2} \mathrm{O}_{3}$ & 35 & 14.23 & 0.21 & 14.23 & 27 & 14.51 & 0.20 & 14.53 \\
\hline $\mathrm{Fe}_{2} \mathrm{O}_{3}$ & 27 & 0.38 & 0.13 & 0.38 & 20 & 2.35 & 0.22 & 2.31 \\
\hline $\mathrm{FeO}$ & 27 & 1.63 & 0.13 & 1.64 & 20 & 5.99 & 0.18 & 6.02 \\
\hline $\mathrm{MnO}$ & 40 & 0.062 & 0.0086 & 0.061 & 30 & 0.15 & 0.011 & 0.15 \\
\hline $\mathrm{MgO}$ & 39 & 0.74 & 0.082 & 0.73 & 32 & 7.72 & 0.14 & 7.74 \\
\hline $\mathrm{CaO}$ & 42 & 2.20 & 0.11 & 2.17 & 30 & 9.27 & 0.18 & 9.24 \\
\hline $\mathrm{Na}_{2} \mathrm{O}$ & 36 & 3.38 & 0.091 & 3.38 & 29 & 2.80 & 0.069 & 2.80 \\
\hline $\mathrm{K}_{2} \mathrm{O}$ & 44 & 3.96 & 0.094 & 3.96 & 32 & 1.43 & 0.092 & 1.44 \\
\hline $\mathrm{P}_{2} \mathrm{O}_{5}$ & 31 & 0.10 & 0.022 & 0.098 & 23 & 0.26 & 0.017 & 0.26 \\
\hline $\mathrm{H}_{2} \mathrm{O}+$ & 24 & 0.53 & 0.14 & 0.52 & 17 & 0.97 & 0.25 & 0.97 \\
\hline $\mathrm{H}_{2} \mathrm{O}-$ & 25 & 0.10 & 0.075 & 0.084 & 18 & 0.97 & 0.14 & 0.97 \\
\hline $\mathrm{CO}_{2}$ & 8 & 0.089 & 0.044 & 0.074 & 6 & 0.19 & 0.048 & 0.19 \\
\hline Sum & & 99.94 & & 99.88 & & 100.19 & & 100.14 \\
\hline $\begin{array}{l}\text { Total } \mathrm{Fe} \\
\text { as } \mathrm{Fe}_{2} \mathrm{O}_{3}\end{array}$ & 38 & 2.19 & 0.15 & 2.17 & 30 & 8.97 & 0.20 & 8.96 \\
\hline Total $\mathrm{H}_{2} \mathrm{O}$ & 26 & 0.64 & 0.15 & 0.64 & 19 & 1.92 & 0.26 & 1.96 \\
\hline
\end{tabular}

$n:$ Number of determinations $\bar{X}:$ Arithmetic mean (\%) $\sigma:$ Standard deviation $\bar{X} c$ : Consensus mean (Arithmetic mean of the analytical values within $2 \sigma)(\%)$

Table 5. Minor constituents (ppm)

\begin{tabular}{|c|c|c|c|c|c|c|}
\hline & JG-1 & & JB-1 & & Method & References \\
\hline Ag & $\begin{aligned} & 0.073 \\
< & 1 \\
< & 0.05 \\
< & 1 \\
& \end{aligned}$ & & $\begin{array}{l}<1{ }^{-} \\
<0.05 \\
2 \\
0.4\end{array}$ & & $\begin{array}{l}\text { OS } \\
\text { OS(DR) } \\
\text { OS } \\
\text { AA } \\
\text { AA }\end{array}$ & $\begin{array}{l}\text { CHAMP (1968) } \\
\text { THOMPSON (1972) } \\
\text { CHAMP and BENDER (1973) } \\
\text { OAKLEY (1973) } \\
\text { GAGNON (1974) }\end{array}$ \\
\hline As & $\begin{array}{r}<300 \\
0.31 \\
0.24 \\
\end{array}$ & (4) & $\begin{array}{r}<300 \\
2.33 \\
1.88\end{array}$ & (4) & $\begin{array}{l}\text { AA } \\
\text { AA } \\
\text { NAA }\end{array}$ & $\begin{array}{l}\text { OAKLEY }(1973) \\
\text { TERASHIMA }(1974 \mathrm{~b}) \\
\text { TERADA }(1974)\end{array}$ \\
\hline B & $\begin{array}{c}5.1 \\
7 \\
12 \\
8 \\
8.7 \\
<10 \\
8.2 \\
\end{array}$ & $\begin{array}{l}\text { (3) } \\
\text { (6) } \\
\text { (av.) }\end{array}$ & $\begin{array}{r}10^{-} \\
16 \\
4 \\
<10^{-}\end{array}$ & (3) & $\begin{array}{l}\text { OS } \\
\text { OS } \\
\text { OS } \\
\text { OS(DR) } \\
\text { Photom. } \\
\text { OS }\end{array}$ & $\begin{array}{l}\text { CHAMP (1968) } \\
\text { IKEDA, K. (1970) } \\
\text { SCHMIDT }(1972) \\
\text { THOMPSON }(1972) \\
\text { ISOZAKI et al. (1973) } \\
\text { CHAMP and BENDER (1973) }\end{array}$ \\
\hline $\mathrm{Ba}$ & $\begin{array}{l}430 \\
470 \\
460 \\
460 \\
430 \\
430 \\
463 \\
525 \\
500\end{array}$ & (3) & $\begin{array}{l}545^{-} \\
455 \\
440 \\
460 \\
485 \\
595 \\
390\end{array}$ & (3) & $\begin{array}{l}\text { OS } \\
\text { ID } \\
\text { AA } \\
\text { OS(DR) } \\
\text { AA } \\
\text { OS } \\
\text { AA } \\
\text { XRF } \\
\text { OS }\end{array}$ & $\begin{array}{l}\text { CHAMP (1968) } \\
\text { NAGASAWA (1971) } \\
\text { TERASHIMA (1971b) } \\
\text { THOMPSON (1972) } \\
\text { RUBESKA (1973) } \\
\text { CHAMP and BENDER (1973) } \\
\text { TERASHIMA (1973b) } \\
\text { MURAD (1973) } \\
\text { BRENDER (1973) }\end{array}$ \\
\hline
\end{tabular}




\begin{tabular}{|c|c|c|c|c|c|c|}
\hline & JG-1 & & JB-1 & & Method & References \\
\hline $\mathbf{B a}$ & $\begin{array}{l}445 \\
603 \\
440 \\
495 \\
473 \\
462\end{array}$ & $\begin{array}{l}\text { (2) } \\
\text { (av.) } \\
\text { (avc.) }\end{array}$ & $\begin{array}{l}501 \\
580 \\
320 \\
515 \\
511 \\
476 \\
490\end{array}$ & $\begin{array}{l}\text { (2) } \\
\text { (av.) } \\
\text { (avc.) }\end{array}$ & $\begin{array}{l}\text { XRF } \\
\text { OS } \\
\text { NAA } \\
\text { AA } \\
\text { ID }\end{array}$ & $\begin{array}{l}\text { GAGNON (1974) } \\
\text { GAGNON (1974) } \\
\text { RANDLE (1974) } \\
\text { RUBESKA (1974) } \\
\text { TANAKA, TS. (1974) }\end{array}$ \\
\hline $\mathrm{Be}$ & $\begin{array}{r}<3 \\
2.4 \\
3.3\end{array}$ & (5) & $1^{-}$ & (5) & $\begin{array}{l}\text { OS } \\
\text { OS } \\
\text { OS } \\
\text { AA }\end{array}$ & $\begin{array}{l}\text { CHAMP }(1968) \\
\text { SCHMIDT }(1972) \\
\text { CHAMP and BENDER (1973) } \\
\text { TERASHIMA }(1973 a)\end{array}$ \\
\hline $\mathrm{Bi}$ & $\begin{array}{rl} & 0.6 \\
<2 & 2 \\
< & 0.5 \\
2.0 & \end{array}$ & & $2_{2}^{-}$ & & $\begin{array}{l}\text { OS } \\
\text { OS(DR) } \\
\text { OS } \\
\text { AA }\end{array}$ & $\begin{array}{l}\text { CHAMP }(1968) \\
\text { THOMPSON (1972) } \\
\text { CHAMP and BENDER (1973) } \\
\text { GAGNON (1974) }\end{array}$ \\
\hline $\mathrm{Br}$ & 0.0 & $8(3)$ & 0.60 & (3) & NAA & AKAIWA et al. (1971) \\
\hline $\mathrm{Cd}$ & $\begin{aligned} & 0.0 \\
< & 0.0 \\
< & 2 \\
< & 0.2\end{aligned}$ & & $\begin{aligned} &- \\
&<.11 \\
&<2 \\
&<0.2\end{aligned}$ & & $\begin{array}{l}\text { AA } \\
\text { AA } \\
\text { OS } \\
\text { AA }\end{array}$ & $\begin{array}{l}\text { YAMADA et al. }(1969) \\
\text { YAMADA et al. (1970) } \\
\text { THOMPSON (1972) } \\
\text { GAGNON }(1974)\end{array}$ \\
\hline $\mathrm{Ce}$ & $\begin{array}{r}42.4 \\
42.8 \\
- \\
41.9 \\
47 \\
44.5 \\
- \\
43.2\end{array}$ & $\begin{array}{l}\text { (3) } \\
(2) \\
\text { (av.) }\end{array}$ & $\begin{array}{l}66.4 \\
\quad- \\
67.9 \\
67.1 \\
65 \\
70 \\
67.1 \\
67.3 \\
\end{array}$ & $\begin{array}{l}\text { (2) } \\
\text { (av.) }\end{array}$ & $\begin{array}{l}\text { ID } \\
\text { ID } \\
\text { ID } \\
\text { NAA } \\
\text { NAA } \\
\text { PAA } \\
\text { ID }\end{array}$ & $\begin{array}{l}\text { NAGASAWA (1971) } \\
\text { NAKAMURA and MASUDA, A. (1971) } \\
\text { TANAKA, TS. and MASUDA, A. (1971) } \\
\text { MASUDA, Y. and YAGI (1971) } \\
\text { RANDLE (1974) } \\
\text { SATO et al. (1974) } \\
\text { TANAKA, TS. (1974) }\end{array}$ \\
\hline $\mathrm{Cl}$ & $\begin{array}{r}200 \\
57 \\
67 \\
54 \\
59 \\
\end{array}$ & $\begin{array}{l}(5) \\
(5) \\
\text { (avc.) }\end{array}$ & $\begin{array}{l}190^{-} \\
167 \\
169 \\
175\end{array}$ & $\begin{array}{l}(5) \\
(5) \\
\text { (av.) }\end{array}$ & $\begin{array}{l}\text { Photom. } \\
\text { NAA } \\
\text { Photom. } \\
\text { Photom. }\end{array}$ & $\begin{array}{l}\text { SEN GUPTA }(1968) \\
\text { AKAIWA et al. (1971) } \\
\text { TERASHIMA (1974a) } \\
\text { TERADA and HIRAKAWA }(1974)\end{array}$ \\
\hline Co & $\begin{array}{c}<20 \\
4.2 \\
- \\
8.8 \\
11 \\
4.0 \\
5 \\
6 \\
<2 \\
16 \\
- \\
4.3 \\
2 \\
28 \\
9 \\
4.0 \\
3.8 \\
4.8\end{array}$ & $\begin{array}{l}(6) \\
(2)\end{array}$ & $\begin{array}{l}{ }^{-}- \\
49 \\
27 \\
37.8 \\
44 \\
38 \\
23 \\
46 \\
31 \\
37.5 \\
35 \\
55 \\
40 \\
40 \\
41.6 \\
42\end{array}$ & $\begin{array}{l}\text { (7) } \\
\text { (4) } \\
(3)\end{array}$ & $\begin{array}{l}\text { OS } \\
\text { AA } \\
\text { AA } \\
\text { AA } \\
\text { AA } \\
\text { NAA } \\
\text { XRF } \\
\text { OS } \\
\text { OS(DR) } \\
\text { AA } \\
\text { OS } \\
\text { AA } \\
\text { AA } \\
\text { AA } \\
\text { XRF } \\
\text { AA } \\
\text { NAA } \\
\text { PAA }\end{array}$ & $\begin{array}{l}\text { CHAMP (1968) } \\
\text { NAGURA and IIDA (1968) } \\
\text { WEIGAND (1970) } \\
\text { TERASHIMA (1971a) } \\
\text { STEELE (1971) } \\
\text { MASUDA, Y. and YAGI (1971) } \\
\text { BEASLEY (1972) } \\
\text { SCHMIDT (1972) } \\
\text { THOMPSON (1972) } \\
\text { OAKLEY (1973) } \\
\text { CHAMP and BENDER (1973) } \\
\text { SCHAFER (1973) } \\
\text { UCHIDA (1973) } \\
\text { GAGNON (1974) } \\
\text { GAGNON (1974) } \\
\text { KAWABE (1974) } \\
\text { RANDLE (1974) } \\
\text { SATO et al. (1974) }\end{array}$ \\
\hline
\end{tabular}




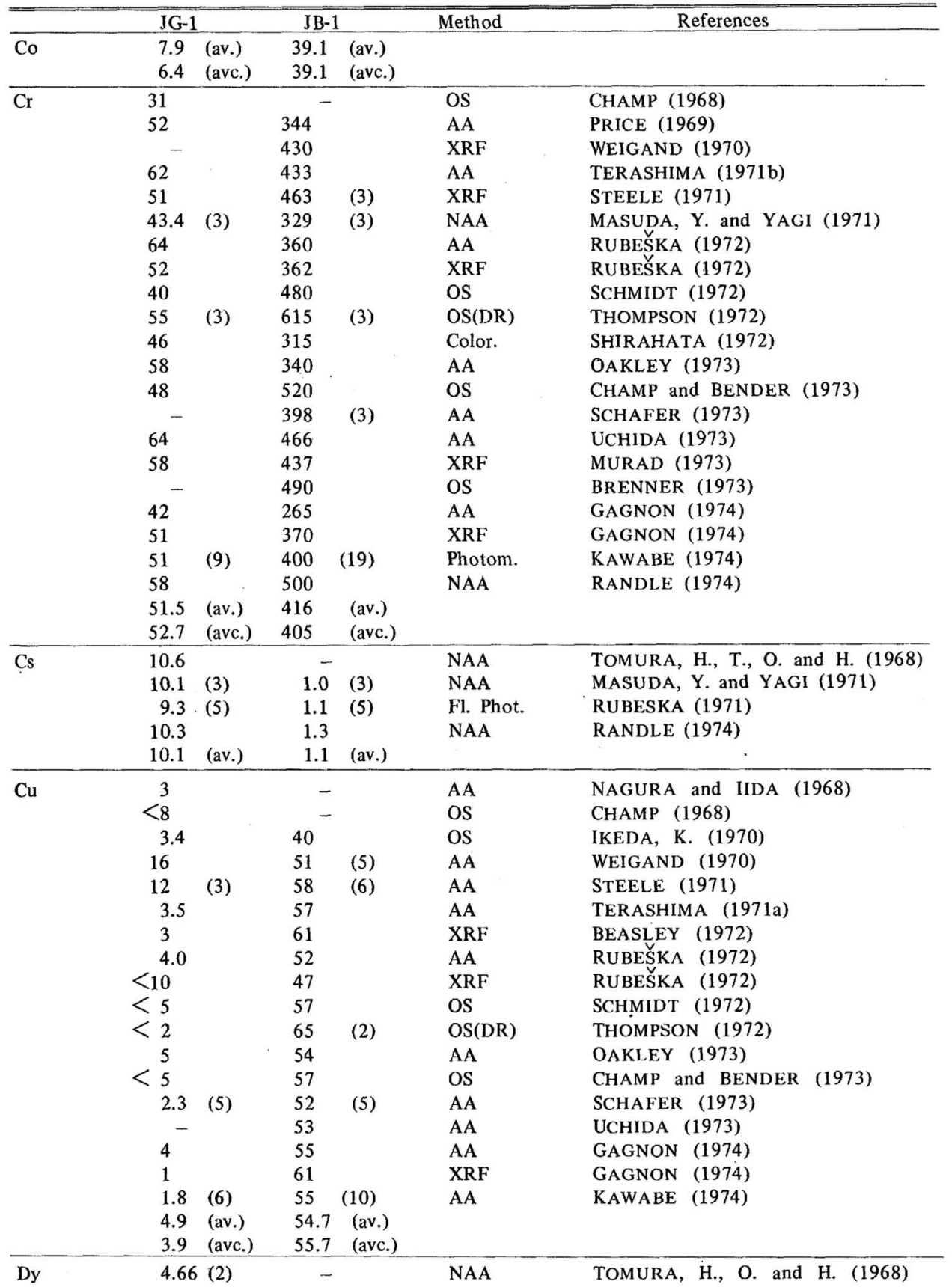




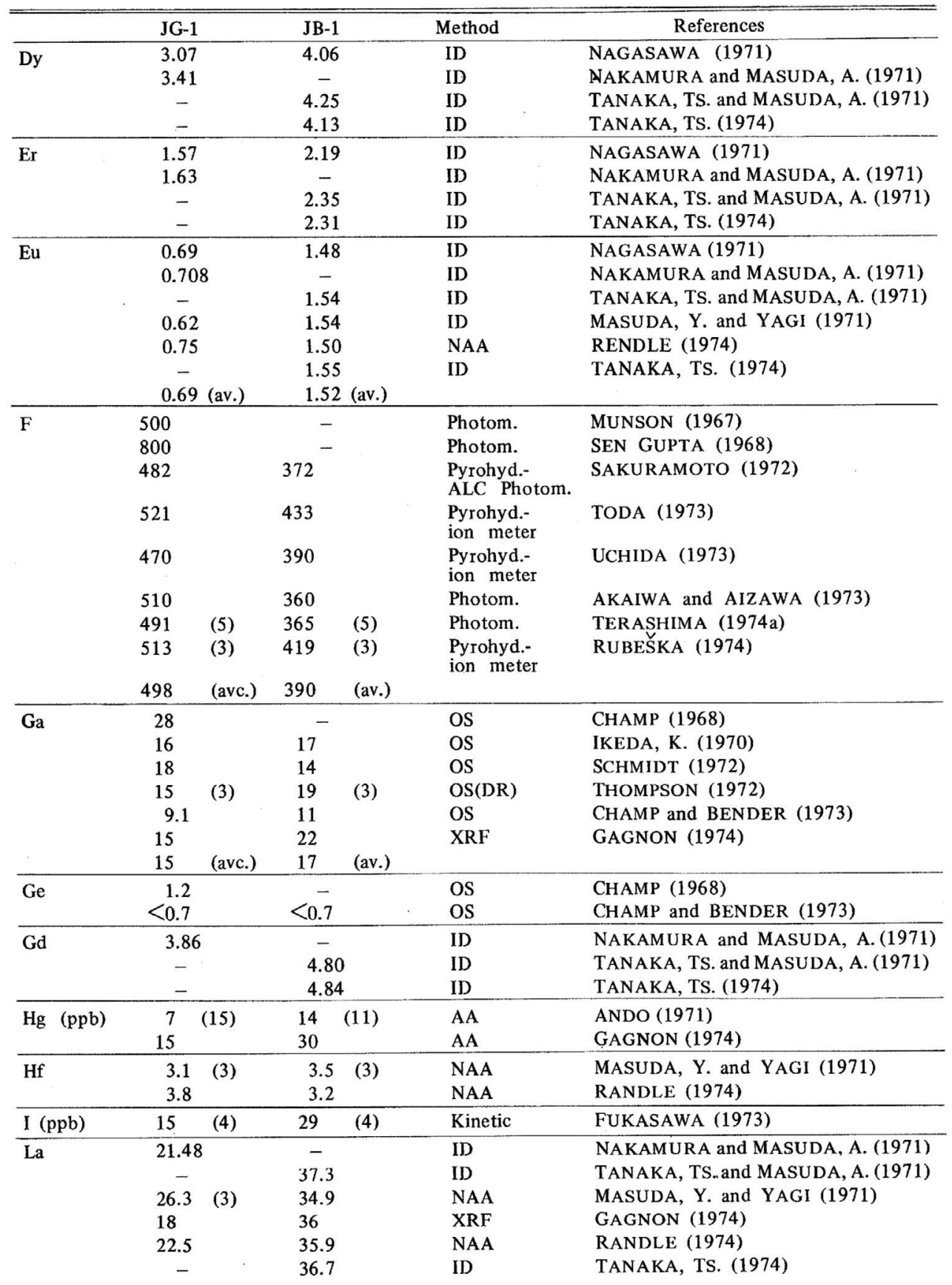




\begin{tabular}{|c|c|c|c|c|c|c|}
\hline & \multicolumn{2}{|l|}{ JG-1 } & \multicolumn{2}{|c|}{ JB-1 } & \multirow[t]{2}{*}{ Method } & \multirow[t]{2}{*}{ References } \\
\hline $\mathrm{La}$ & 22.1 & (av.) & 36.2 & (av.) & & \\
\hline \multirow[t]{9}{*}{$\mathrm{Li}$} & 99.3 & & 7.67 & & AA & PRICE (1969) \\
\hline & 106 & & 12 & & AA & WEIGAND (1970) \\
\hline & 96 & & 11 & & AA & TERASHIMA (1971a) \\
\hline & - & & 10 & (5) & AA & STEELE (1971) \\
\hline & 84 & & 11.7 & & AA & RUBESKA (1972) \\
\hline & 95 & (3) & 16 & (3) & OS(DR) & THOMPSON (1972) \\
\hline & 83 & & 12 & & $\mathrm{AA}$ & OAKLEY (1973) \\
\hline & - & & 11 & (6) & AA & SCHAFER (1973) \\
\hline & 94 & (av.) & 11.4 & (av.) & & \\
\hline \multirow[t]{7}{*}{$\mathrm{Lu}$} & \multicolumn{2}{|c|}{0.238} & \multicolumn{2}{|c|}{0.302} & ID & NAGASAWA (1971) \\
\hline & \multirow{2}{*}{\multicolumn{2}{|c|}{$\begin{array}{c}0.22 \\
-\end{array}$}} & \multicolumn{2}{|c|}{-} & ID & NAKAMURA and MASUDA, A. (1971) \\
\hline & & & \multicolumn{2}{|c|}{0.31} & ID & TANAKA, TS. and MASUDA, A. (1971) \\
\hline & \multicolumn{2}{|l|}{0.52} & \multicolumn{2}{|c|}{0.29} & NAA & MASUDA, Y. and YAGI (1971) \\
\hline & \multicolumn{2}{|l|}{0.45} & \multicolumn{2}{|c|}{0.33} & NAA & RANDLE (1974) \\
\hline & \multirow{2}{*}{\multicolumn{2}{|c|}{-}} & \multirow{2}{*}{\multicolumn{2}{|c|}{$\begin{array}{l}0.307 \\
0.31 \text { (av.) }\end{array}$}} & ID & TANAKA, TS. (1974) \\
\hline & & & & & & \\
\hline \multirow[t]{6}{*}{ Mo } & \multicolumn{2}{|l|}{2.3} & - & & OS & CHAMP (1968) \\
\hline & 2.2 & & 16 & & OS & IKEDA, K. (1970) \\
\hline & - & & 30 & & OS & SCHMIDT (1972) \\
\hline & 2 & & 22 & (3) & OS(DR) & THOMPSON (1972) \\
\hline & 1.0 & & 8 & & OS & CHAMP and BENDER (1973) \\
\hline & 2 & & 48 & & AA & GAGNON (1974) \\
\hline $\mathrm{Nb}$ & - & & 20 & & OS & SCHMIDT (1972) \\
\hline & 11.5 & (2) & 15 & (2) & PAA & SATO et al. (1974) \\
\hline & 13 & & 27 & & $\mathrm{XRF}$ & GAGNON (1974) \\
\hline Nd & 18.5 & & 20.8 & & ID & NAGASAWA (1971) \\
\hline & 20.58 & & - & & ID & NAKAMURA and MASUDA, A. (1971) \\
\hline & - & & 27.0 & & ID & TANAKA, TS. and MASUDA, A. (1971) \\
\hline & $<30$ & & $<30$ & & NAA & RANDLE (1974) \\
\hline & - & & 26.6 & & ID & TANAKA, TS. (1974) \\
\hline $\mathrm{Ni}$ & $<20$ & & - & & OS & CHAMP (1968) \\
\hline & 6.1 & & - & & $\mathrm{AA}$ & NAGURA and IIDA (1968) \\
\hline & - & & 137 & & $\mathrm{XRF}$ & WEIGAND $(1970)$ \\
\hline & 7.0 & & 148 & & AA & TERASHIMA (1971a) \\
\hline & 14 & (2) & 132 & (4) & $\mathrm{XRF}$ & STEELE (1971) \\
\hline & 6 & & 143 & & $\mathrm{XRF}$ & BEASLEY (1972) \\
\hline & $<30$ & & 145 & & AA & RUBEŠKA (1972) \\
\hline & $<10$ & & 120 & & $\mathrm{XRF}$ & RUBESKA (1972) \\
\hline & 8 & & 120 & & OS & SCHMIDT (1972) \\
\hline & 6 & (3) & 110 & (3) & OS(DR) & THOMPSON (1972) \\
\hline & 12 & & 139 & & AA & OAKLEY (1973) \\
\hline & - & & 130 & & OS & CHAMP and BENDER (1973) \\
\hline & 6 & (4) & 129 & (5) & AA & SCHAFER (1973) \\
\hline & 6 & & 122 & & AA & UCHIDA (1973) \\
\hline & 20 & & 146 & & $\mathrm{AA}$ & GAGNON (1974) \\
\hline & 9 & & 88 & & XRF & GAGNON (1974) \\
\hline & 11.5 & (2) & 166 & (2) & PAA & KATO et al. (1974) \\
\hline & 6.3 & (6) & 140 & (10) & $\mathrm{AA}$ & KAWABE (1974) \\
\hline & 9.1 & (av.) & 132 & (av.) & & \\
\hline & 8.2 & (avc.) & 135 & (avc.) & & \\
\hline $\mathrm{O}(\%)$ & 49.40 & & 45.66 & & NAA & HUYSMANS (1973) \\
\hline
\end{tabular}




\begin{tabular}{|c|c|c|c|c|c|c|}
\hline & \multicolumn{2}{|c|}{ JG-1 } & \multicolumn{2}{|l|}{ JB-1 } & Method & References \\
\hline Os & \multicolumn{2}{|c|}{$0.0027(5)$} & \multicolumn{2}{|c|}{$0.0019(5)$} & NAA & TERADA et al. (1972) \\
\hline \multirow[t]{20}{*}{$\mathrm{Pb}$} & \multicolumn{2}{|c|}{24} & \multicolumn{2}{|c|}{-} & AA & NAGURA and IIDA (1968) \\
\hline & \multicolumn{2}{|l|}{23} & \multicolumn{2}{|l|}{ - } & OS & СНАMP $(1968)$ \\
\hline & \multicolumn{2}{|l|}{24.9} & \multicolumn{2}{|l|}{-} & AA & TANAKA, TA. and IIDA (1969) \\
\hline & \multicolumn{2}{|l|}{21} & \multicolumn{2}{|l|}{16} & AA & PRICE (1969) \\
\hline & \multicolumn{2}{|l|}{28} & \multicolumn{2}{|l|}{14.5} & OS. & IKEDA, K. (1970) \\
\hline & \multicolumn{2}{|l|}{24} & \multicolumn{2}{|l|}{11} & AA & TERASHIMA (1971a) \\
\hline & \multicolumn{2}{|l|}{26} & \multicolumn{2}{|l|}{7.6} & $\mathrm{XRF}$ & BEASLEY (1972) \\
\hline & \multicolumn{2}{|l|}{27} & 9.3 & & AA & RUBEŠKA (1972) \\
\hline & 23 & & 10 & & $\mathrm{XRF}$ & RUBEŠKA (1972) \\
\hline & 20 & & 9 & & OS & SCHMIDT (1972) \\
\hline & 32 & (3) & 12 & (3) & $\mathrm{OS}(\mathrm{DR})$ & THOMPSON (1972) \\
\hline & 28 & & 23 & & $\mathrm{AA}$ & OAKLEY (1973) \\
\hline & 22 & & 6.7 & & OS & CHAMP and BENDER (1973) \\
\hline & 32 & & 15 & & AA & UCHIDA (1973) \\
\hline & 27 & & 5 & & XRF & MURAD (1973) \\
\hline & 25 & & - & & AA & GAGNON (1974) \\
\hline & 33 & & 15 & & XRF & GAGNON (1974) \\
\hline & 26 & (6) & 7 & $(10)$ & $\mathrm{AA}$ & KAWABE (1974) \\
\hline & 25.9 & (av.) & 11.5 & (avc.) & & 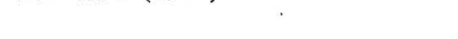 \\
\hline & 25.9 & (avc.) & 10.6 & (avc.) & & \\
\hline $\mathrm{Ra}(\mu \mu \mathrm{g} / \mathrm{g})$ & 1.24 & & 0.72 & & $\mathrm{Rn}$ & NISHIMURA (1969) \\
\hline $\mathrm{Rb}$ & 185 & & - & & XRF & HATTORI and SHIBATA (1967) \\
\hline & 187 & (2) & - & & NAA & TOMURA, H., T., O. and H. (1968) \\
\hline & 207 & (2) & 44.9 & (2) & ID & TANIGUCHI and OKADA (1968) \\
\hline & 183 & & 42.4 & & ID & HAYASE (1968) \\
\hline & 181 & (4) & 40.7 & (3) & ID & TAMURA (1968) \\
\hline & 188.2 & & 43.4 & & ID & UEDA, N. (1968) \\
\hline & 180 & (3) & 40 & & ID & TAKEUCHI and SHIMA, MAS. (1968) \\
\hline & 168 & & 41 & (2) & NAA & TAKEUCHI and SHIMA, MAS. (1968) \\
\hline & 184.8 & (2) & - & & ID & YAMAGUCHI (1968) \\
\hline & 187 & & 34 & & AA & PRICE (1969) \\
\hline & 187 & (4) & 41 & (3) & $\mathrm{AA}$ & UEDA, Y. et al. (1969) \\
\hline & 202 & & 39 & & XRF & HATTORI and SHIBATA (1969) \\
\hline & 181.8 & (3) & - & & ID & SATO, S. et al. (1969) \\
\hline & 185 & & 41 & & $\mathrm{XRF}$ & KURASAWA $(1970)$ \\
\hline & 178 & (3) & 39.4 & (3) & ID & SHIBATA et al. (1970) \\
\hline & - & & 42 & & AA & WEIGAND (1970) \\
\hline & - & & 40 & (11) & AA & STEELE (1971) \\
\hline & 181 & & 48 & & AA & GOVINDARAJU et al. (1971) \\
\hline & 179.5 & & - & & ID & ISHIZAKA (1971) \\
\hline & 179 & & 38.9 & & ID & SHIBATA and ADACHI (1972) \\
\hline & 179 & & 41.5 & & XRF & BEASLEY (1972) \\
\hline & 188 & & 44 & & $\mathrm{AA}$ & RUBEŠKA (1972) \\
\hline & 174 & & 40 & & XRF & RUBEŠKA (1972) \\
\hline & 180 & (3) & 55 & (3) & $\mathrm{OS}(\mathrm{DR})$ & THOMPSON (1972) \\
\hline & 171 & & 44 & & AA & OAKLEY (1973) \\
\hline & 182 & (3) & 40 & (3) & AA & TERASHIMA (1973b) \\
\hline & 184 & (3) & 41 & (3) & F1. Phot. & TERASHIMA (1973b) \\
\hline & 181 & & 38 & & XRF & MURAD (1973) \\
\hline & 187 & & 41 & & XRF & GAGNON (1974) \\
\hline
\end{tabular}




\begin{tabular}{|c|c|c|c|c|c|c|}
\hline \multirow{2}{*}{$\mathrm{Rb}$} & \multicolumn{2}{|c|}{ JG-1 } & \multicolumn{2}{|l|}{ JB-1 } & \multirow{2}{*}{ Method } & References \\
\hline & - & & 39 & & & FUJIMAKI (1974) \\
\hline & 178.9 & & - & & ID & MAHON (1974) \\
\hline & 183 & & $<45$ & & NAA & RANDLE (1974) \\
\hline & 175.5 & (2) & 46 & (2) & PAA & SATO et al. (1974) \\
\hline & 178.9 & & - & & ID & SHIRAHASE (1974) \\
\hline & 182.8 & (av.) & 41.7 & (av.) & & \\
\hline & 181.3 & (avc.) & 41.2 & (avc.) & & \\
\hline \multirow[t]{2}{*}{$\mathrm{S}$} & \multicolumn{2}{|l|}{-} & 50 & (3) & Comb. Vol. & BOUVIER (1970) \\
\hline & 23 & (6) & 40 & (2) & Photom. & OZAWA et al. (1972) \\
\hline \multirow[t]{3}{*}{$\mathrm{Sb}$} & \multicolumn{2}{|c|}{$<2.0$} & \multicolumn{2}{|l|}{$<2.0$} & $\mathrm{AA}$ & GAGNON (1974) \\
\hline & \multicolumn{2}{|c|}{0.10} & \multicolumn{2}{|l|}{0.24} & $\mathrm{AA}$ & TERASHIMA (1974b) \\
\hline & \multicolumn{2}{|l|}{$<0.6$} & \multicolumn{2}{|l|}{0.22} & NAA & RANDLE (1974) \\
\hline \multirow[t]{4}{*}{ Sc } & \multicolumn{2}{|l|}{$<20$} & \multicolumn{2}{|l|}{-} & OS & CHAMP (1968) \\
\hline & \multicolumn{2}{|l|}{6.44} & \multicolumn{2}{|l|}{25.6} & NAA & MASUDA, Y. and YAGI (1971) \\
\hline & \multicolumn{2}{|c|}{8.0} & 32 & & OS & CHAMP and BENDER (1973) \\
\hline & 6.54 & & 27.5 & & NAA & RANDLE (1974) \\
\hline Se & 0.00 & & 0.02 & & NAA & TERADA et al. (1974) \\
\hline $\mathrm{Sm}$ & 4.15 & & 4.93 & & ID & NAGASAWA (1971) \\
\hline & 4.48 & & - & & ID & NAKAMURA and MASUDA, A. (1971) \\
\hline & - & & 5.1 & & ID & TANAKA, TS, and MASUDA, A. (1971) \\
\hline & 4.51 & (3) & 4.31 & (3) & NAA & MASUDA, Y. and YAGI (1971) \\
\hline & 5.4 & & 4.7 & & NAA & RANDLE (1974) \\
\hline & - & & 5.16 & & ID & TANAKA, TS. (1974) \\
\hline & 4.6 & (av.) & 4.8 & (av.) & & \\
\hline $\mathrm{Sn}$ & 3 & & - & & OS & CHAMP (1968) \\
\hline & 3 & & 2.3 & & OS & IKEDA, K. (1970) \\
\hline & - & & 4 & & OS & SCHMIDT (1972) \\
\hline & 3.1 & & 1.4 & & OS & CHAMP and BENDER (1973) \\
\hline & 4.1 & (3) & 2.1 & (3) & AA & TERASHIMA (1974c) \\
\hline $\mathrm{Sr}$ & 182 & (4) & 425 & (4) & ID & TAMURA (1968) \\
\hline & 182.5 & & 447 & & ID & TAKEUCHI and SHIMA, MAS. (1968) \\
\hline & 187 & (2) & 466 & & NAA & TAKEUCHI and SHIMA, MAS. (1968) \\
\hline & 185.1 & (3) & 455.1 & (2) & ID & UEDA, N. (1968) \\
\hline & 180.5 & (2) & 424 & (2) & ID & TANIGUCHI and OKADA (1968) \\
\hline & 188.1 & (3) & - & & ID & YAMAGUCHI (1968) \\
\hline & 160 & & - & & OS & CHAMP (1968) \\
\hline & 184.9 & (3) & - & & ID & SATO, S. et al. (1969) \\
\hline & 170 & & 547 & & AA & PRICE (1969) \\
\hline & 194 & & 417 & & XRF & HATTORI and SHIBATA (1969) \\
\hline & 186 & (2) & 448 & & ID & SHIBATA et al. (1970) \\
\hline & - & & 450 & & $\mathrm{XRF}$ & WEIGAND (1970) \\
\hline & 181 & & - & & ID & NAGASAWA (1971) \\
\hline & 191 & & 424 & & $\mathrm{AA}$ & TERASHIMA (1971a) \\
\hline & - & & 435 & & XRF & STEELE (1971) \\
\hline & 186.8 & & - & & ID & ISHIZAKA (1971) \\
\hline & 187.5 & & 448 & & ID & SHIBATA and ADACHI (1972) \\
\hline & 185 & & 450 & & XRF & BEASLEY (1972) \\
\hline & 185 & & 400 & & $\mathrm{AA}$ & RUBEŠKA (1972) \\
\hline & 180 & & 410 & & XRF & RUBESKKA (1972) \\
\hline & 165 & (3) & 390 & (3) & $\mathrm{OS}(\mathrm{DR})$ & THOMPSON (1972) \\
\hline & 195 & & 413 & & AA & OAKLEY (1973) \\
\hline
\end{tabular}




\begin{tabular}{|c|c|c|c|c|c|c|}
\hline & $J G-1$ & & JB-1 & & Method & References \\
\hline \multirow[t]{11}{*}{$\mathrm{Sr}$} & 180 & & 450 & & OS & CHAMP and BENDER (1973) \\
\hline & 199 & & 450 & & XRF & MURAD (1973) \\
\hline & 110 & & 455 & & OS & BRENNER (1973) \\
\hline & - & & 442 & & ID & FUJIMAKI (1974) \\
\hline & 185.1 & & 445 & & ID & SHIRAHASE (1974) \\
\hline & 185.8 & & - & & ID & MAHON (1974) \\
\hline & 199 & & 428 & & $\mathrm{XRF}$ & GAGNON (1974) \\
\hline & 182 & & 618 & & OS & GAGNON (1974) \\
\hline & 182.5 & & 447.5 & & PAA & SATO et al. (1974) \\
\hline & 181.4 & (av.) & 447.0 & (av.) & & \\
\hline & 184.1 & (avc.) & 435.2 & (avc.) & & \\
\hline Ta & 2.9 & & 4.4 & & NAA & RANDLE (1974) \\
\hline $\mathrm{Tb}$ & 0.43 & (3) & 0.47 & & NAA & MASUDA, Y. and YAGI (1971) \\
\hline \multirow[t]{13}{*}{ Th } & 13.4 & & - & & Photom. & MIYAKE and SUGIYAMA (1967) \\
\hline & 14 & & - & & Photom. & SEKINE and MOCHIZUKI (1968) \\
\hline & 14 & & - & & $\gamma$ cntg. & SHIMA, MAKOTO (1968) \\
\hline & 13.2 & & 9.2 & & $\gamma$ cntg. & KANAYA $(1970)$ \\
\hline & 11.5 & & 7.8 & & $\alpha$ cntg. & MIYAKE et al. (1970) \\
\hline & 13.7 & (3) & 11.8 & & NAA & MASUDA, Y. and YAGI (1971) \\
\hline & 13.4 & & 8.95 & & $\gamma \mathrm{cntg}$ & KANAYA $(1971)$ \\
\hline & 13 & & 9 & & XRF & BEASLEY (1972) \\
\hline & 15.6 & & 9.8 & & $\gamma \mathrm{cntg}$ & ADAMS $(1972)$ \\
\hline & 13.1 & (2) & 9.04 & & Photom. & KIRIYAMA and KURODA (1974) \\
\hline & 13.8 & & 8.9 & & NAA & RANDLE (1974) \\
\hline & 13.5 & (av.) & 9.3 & (av.) & & \\
\hline & 13.5 & (avc.) & 9.0 & (avc.) & & \\
\hline \multirow[t]{3}{*}{$\mathrm{Tl}$} & 2.3 & & - & & $\overline{\mathrm{OS}}$ & CHAMP (1968) \\
\hline & 2.5 & & - & & OS & IKEDA, K. (1970) \\
\hline & 1.0 & & - & & OS & CHAMP and BENDER (1973) \\
\hline \multirow[t]{17}{*}{$\mathrm{U}$} & 3.1 & & - & & Photom. & MIYAKE and SUGIMURA (1967) \\
\hline & 3.0 & & 1.5 & & Fluo. & SEKINE and MOCHIZUKI (1968) \\
\hline & 3.1 & & - & & $\gamma$ cntg. & SHIMA, MAKOTO (1968) \\
\hline & 3.7 & & - & & eq. $U(R a)$ & NISHIMURA (1969) \\
\hline & 3.5 & & 2.0 & & FT & HASHIMOTO (1969) \\
\hline & 3.3 & & 1.8 & & FT & NISHIMURA (1970) \\
\hline & 3.3 & & - & & NAA & NISHIMURA (1970) \\
\hline & 3.2 & & 1.7 & & $\alpha$ cntg. & MIYAKE et al. (1970) \\
\hline & - & & 1.9 & & $\gamma$ cntg. & KANAYA $(1970)$ \\
\hline & 4.67 & & 1.72 & & $\gamma$ cntg. & KANAYA (1971) \\
\hline & 5.0 & & 1.6 & & $\gamma$ cntg. & ADAMS (1972) \\
\hline & 2 & & 2 & & $\mathrm{XRF}$ & BEASLEY (1972) \\
\hline & 3.0 & & 1.85 & & NAA & IKEDA, N. (1972) \\
\hline & 4.2 & & 1.84 & & Photom. & KIRIYA and KURODA (1974) \\
\hline & 2.5 & & - & & Chromatg. & GAGNON (1974) \\
\hline & 3.4 & (av.) & 1.8 & (av.) & & \\
\hline & 3.3 & (avc.) & 1.8 & (avc.) & & \\
\hline \multirow[t]{5}{*}{$\mathrm{V}$} & 25 & & - & & OS & CHAMP (1968) \\
\hline & 21 & & 390 & & OS & IKEDA (1970) \\
\hline & 25 & & 200 & & Photom. & TERASHIMA (1970) \\
\hline & $<25$ & & 223 & & AA & RUBEŠKA (1972) \\
\hline & - & & 205 & & os & SCHMIDT (1972) \\
\hline
\end{tabular}




\begin{tabular}{|c|c|c|c|c|c|c|}
\hline \multirow[b]{2}{*}{$\mathrm{V}$} & \multicolumn{2}{|l|}{ JG-1 } & \multicolumn{2}{|l|}{ JB-1 } & \multirow{2}{*}{$\frac{\text { Method }}{\text { OS(DR) }}$} & \multirow{2}{*}{$\frac{\text { References }}{\text { THOMPSON (1972) }}$} \\
\hline & 95 & (3) & 170 & (3) & & \\
\hline & 28 & & 240 & & OS & CHAMP and BENDER (1973) \\
\hline & 25 & (5) & 227 & (6) & AA & SCHAFER (1973) \\
\hline & 21 & (5) & 215 & (5) & AA & TERASHIMA (1973a) \\
\hline & 22 & & 220 & & os & BRENNER (1973) \\
\hline & 25 & & 228 & & AA & GAGNON (1974) \\
\hline & 21 & & 217 & & XRF & GAGNON (1974) \\
\hline & 23.3 & & 178 & & Photom. & AKAIWA et al. (1974) \\
\hline & 30 & (av.) & 226 & (avc.) & & , \\
\hline & 24 & (avc.) & 211 & (avc.) & & \\
\hline W & - & & 14 & & $\mathrm{XRF}$ & GAGNON (1974) \\
\hline \multirow[t]{6}{*}{$\mathrm{Y}$} & 30 & & - & & OS & CHAMP (1968) \\
\hline & 32 & & 26 & & $\mathrm{XRF}$ & BEASLEY (1972) \\
\hline & - & & 24 & (3) & OS(DR) & THOMPSON (1972) \\
\hline & $<30$ & & $<30$ & & OS & CHAMP and BENDER (1973) \\
\hline & 30.5 & (2) & 26.5 & $(2)$ & PAA & SATO et al. (1974) \\
\hline & 30.8 & (av.) & 25.5 & (av.) & & \\
\hline \multirow[t]{8}{*}{$\mathrm{Yb}$} & $<10$ & & - & & OS & CHAMP (1968) \\
\hline & 1.52 & & 2.01 & & ID & NAGASAWA (1971) \\
\hline & 1.48 & & - & & ID & NAKAMURA and MASUDA, A. (1971) \\
\hline & - & & 2.15 & & ID & TANAKA, TS. and MASUDA, A. (1971) \\
\hline & 2.50 & & 2.18 & & NAA & MASUDA, Y. and YAGI (1971) \\
\hline & $<4$ & & $<4$ & & os & CHAMP and BENDER (1973) \\
\hline & 2.5 & & 1.2 & & NAA & RANDLE (1974) \\
\hline & - & & 2.13 & & ID & TANAKA, TS. (1974) \\
\hline \multirow[t]{18}{*}{$\mathrm{Zn}$} & 35 & & - & & OS & CHAMP (1968) \\
\hline & 30 & & 71.5 & & AA & PRICE (1969) \\
\hline & 46 & & 95 & & AA & KATO, Y. (1970) \\
\hline & - & & 86 & & AA & WEIGAND (1970) \\
\hline & 35 & & 83 & & AA & TERASHIMA (1971a) \\
\hline & 34 & & 78 & & AA & STEELE (1971) \\
\hline & 41 & & 91 & & XRF & BEASLEY (1972) \\
\hline & 49 & & 90 & & AA & RUBEŠKA (1972) \\
\hline & 45 & & 86 & & XRF & RUBEŠKA (1972) \\
\hline & 35 & (3) & 170 & (3) & $\mathrm{OS}(\mathrm{DR})$ & THOMPSON (1972) \\
\hline & 39 & & 82 & & $\mathrm{AA}$ & OAKLEY (1973) \\
\hline & 38 & & 79 & & OS & CHAMP and BENDER (1973) \\
\hline & 40 & & 84 & & AA & UCHIDA (1973) \\
\hline & 44 & & 74 & & $\mathrm{XRF}$ & MURAD (1973) \\
\hline & 45 & & 77 & & AA & GAGNON (1974) \\
\hline & 48 & & 98 & & $\mathrm{XRF}$ & GAGNON (1974) \\
\hline & 40 & (av.) & 90 & (av.) & & \\
\hline & 40 & (avc.) & 84 & (avc.) & & \\
\hline \multirow[t]{8}{*}{$\mathrm{Zr}$} & 74 & & - & & OS & CHAMP (1968) \\
\hline & - & & 148 & & XRF & WEIGAND (1970) \\
\hline & - & & 163 & & XRF & STEELE (1971) \\
\hline & 106 & & 139 & & XRF & BEASLEY (1972) \\
\hline & 75 & (3) & 135 & (3) & OS(DR) & THOMPSON (1972) \\
\hline & 140 & & 140 & & OS & CHAMP and BENDER (1973) \\
\hline & 122 & & 140 & & XRF & MURAD (1973) \\
\hline & 97.8 & & 151 & & Photom. & KIRIYAMA and KURODA (1974) \\
\hline
\end{tabular}




\begin{tabular}{|c|c|c|c|c|c|c|}
\hline & \multicolumn{2}{|c|}{ JG-1 } & \multicolumn{2}{|l|}{ JB-1 } & Method & References \\
\hline \multirow[t]{5}{*}{$\mathrm{Zr}$} & 115 & & 215 & & XRF & GAGNON (1974) \\
\hline & 160 & & 360 & & NAA & RANDLE (1974) \\
\hline & 106 & (2) & 143.5 & (2) & PAA & SATO et al. (1974) \\
\hline & 111 & (av.) & 173 & (av.) & & \\
\hline & 111 & (avc.) & 153 & (avc.). & & \\
\hline
\end{tabular}

Table 6. Strontium isotopic ratios

\begin{tabular}{|c|c|c|c|c|c|}
\hline & JG-1 & & JB-1 & Method & References \\
\hline \multirow{13}{*}{$\begin{array}{c}{ }^{87} \mathrm{Sr} /^{86} \mathrm{Sr} \\
\text { norm. }\end{array}$} & 0.7115 & (2) & 0.7047 & $\mathrm{M}$ & TAKEUCHI and SHIMA, MAS. (1968) \\
\hline & 0.7116 & (2) & 0.7047 & M & UEDA, N. (1968) \\
\hline & 0.7117 & (3) & 0.7053 & M & YAMAGUCHI (1968) \\
\hline & 0.7117 & (2) & - & M & SATO et al. (1969) \\
\hline & 0.7113 & (2) & 0.7043 & M & SHIBATA $(1970)$ \\
\hline & 0.7118 & & $0.7056^{*}$ & M & KURASAWA (1970) \\
\hline & 0.7115 & & - & M & ISHIZAKA (1971) \\
\hline & 0.7114 & (2) & 0.7043 & M & SHIBATA and ADACHI (1972) \\
\hline & 0.7108 & & $0.7043 \quad(2)$ & M & SHIRAHASE (1974) \\
\hline & 0.7111 & (2) & - & M & MAHON (1974) \\
\hline & - & & 0.7046 & M & KURASAWA (1974) \\
\hline & - & & 0.7044 & M & FUJIMAKI (1974) \\
\hline & 0.7114 & (av.) & 0.7046 (av.) & & \\
\hline
\end{tabular}

* : This is omitted from the calculation of the average as it has been superseded by the revised value (KURASAWA, 1974).

\section{REFERENCES}

ADAMS, J. A. S. (1972) Private communication, Rice University, Houston, Texas, U.S.A.

AKAIWA, H., TAZIMA, E. and TODOKORO, A. (1971) Private communication, Gunma University, Kiriu, Gunma, Japan.

AKAIWA, H. and AIZAWA, S. (1973) Behavior of fluorine during sedimentary processes - Fluorine contents of sedimentary rocks from Tomioka, southern Gunma Prefecture, Japan Geochem. J. 7, 1-9.

AKAIWA, H., KAWAMOTO, H. and KONDO, H. (1974) Extractive spectrophotometric determination of vanadium (V) by using the combination of 1-butanol and PAR. Japan Analyst (Bunseki Kagaku) 23, 402-406 (in Japanese with English abstract).

ANDO, A. (1967) A new silicate rock standard, JG-1 issued from the Geological Survey of Japan. Geochem. J. 1, 155.

ANDo, A. (1971) Private communication, Geological Survey of Japan, Kawasaki, Japan.

ANDO, A., KURASAWA, H., OHMORI, T. and TAKEDA, E. (1971) 1971 compilation of data on rock standards JG-1 and JB-1 issued from the Geological Survey of Japan. Geochem. J. 5, 151-164.

BEASLEY, P. H. (1972) Private communication, Department of Geophysics and Geochemistry, The Australian National University, Canberra, Australia.

Bouvier, J. L. (1970) Private communication, Geological Survey of Canada, Ottawa, Canada. BRENNER, I. B. (1973) Private communication, Geological Survey of Israel, Jerusalem, Israel. Champ, W. H. (1968) Private communication, Geological Survey of Canada, Ottawa, Canada. 
Champ, W. H. and Bender, G. P. (1973) Private communication, Geological Survey of Canada, Ottawa, Canada.

FLANAGAN, F. J. (1973) 1972 values for international geochemical reference samples. Geochim. Cosmochim. Acta 37, 1189-1200.

FrIESE, D. C. G. (1972) Quoted in SCHMIDT, K. (1972).

FuJIMAKI, H. (1974) Private communication, Geological Institute, University of Tokyo, Tokyo, Japan.

FuKasAWA, T. (1973) Private communication, Faculty of Engineering, Yamanashi University, Kofu, Japan.

Gagnon, J. (1974) Private communication, Service Analyse et Contrôle, Comlexe Scientifique, Ste-Foy, Qué. Canada.

Govindaraju, K., Mevelle, G. and Chouard, C. (1971) Direct atomic absorption determination of rubidium on pulverized silicate rock samples. Chem. Geol. 8, 131-137.

HASHIMOTO, T. (1969) Private communication, Kyoto University, Kyoto, Japan.

HATTORI, H. and ShiBATA, K. (1967) Private communication, Geological Survey of Japan, Kawasaki, Japan.

HATTORI, H. and ShibATA, K. (1969) Quantitative analysis of Rb and $\mathrm{Sr}$ in rocks by X-ray fluorescence spectrometry. Bull. Geol. Surv. Japan 20, 51-76 (in Japanese with English abstract).

HAYASE, I. (1968) Private communication, Kyoto University, Kyoto, Japan.

HAYATA, T. (1974) Chemical analysis of geochemical standard sample JB-1 (1). Nagasaki-ken Chigakukaishi No. 21, 19-21 (in Japanese).

HUYSMANS, K. (1973) Private communication, Institut voor Nucleaire Wetenschappen, Ruksuniversiteit, Gent, Belgium.

HUYSMANS, K. (1974) Private communication, Institut voor Nucleaire Wetenschappen, Ruksuniversiteit, Gent, Belgium.

IKEDA, K. (1970) Private communication, Geological Survey of Japan, Kawasaki, Japan.

IKEDA, N. (1972) Private communication, Tokyo Kyoiku University, Tokyo, Japan.

ISHIZAKA, K. (1971) A Rb-Sr isotopic study of the Ibaragi granitic complex, Osaka, Japan. $J$. Geol. Soc. Japan 77, 731-740.

ISOZAKI, A., YoShIDA, M., UTSUMI, S. and IWASAKI, I. (1973) The boron content of volcanic rocks in Japan. Nippon Kagaku Kaishi 22, 1896-1904 (in Japanese with English abstract).

KanaYA, H. (1970, 1971) Private communication, Geological Survey of Japan, Kawasaki, Japan.

Kato, T., Kitazume, E. and SuzUKI, N. (1974) Private communication, Tohoku University, Sendai, Japan.

KATO, Y. (1970) Total $\mathrm{Fe}_{2} \mathrm{O}_{3}$ and $\mathrm{Zn}$ contents in the geochemical standards JB-1 and JG-1 determined by atomic absorption spectrometry. J. Jap. Assoc. Min. Pet. Econ. Geol. 64, 31-34 (in Japanese with English abstract).

KAWABE, I. (1974) Transition metal contents of Paleozoic geosynclinal basalts in Southwest Japan and their geological significance. J. Geol. Soc. Japan 80, 539-554 (in Japanese with English abstract).

KIRIYAMA, T. and KURODA, R. (1974) Ion-exchange separation and spectrophotometric determination of zirconium, thorium and uranium in silicate rocks with arsenazo III. Anal. Chim. Acta 71, 375-381.

KuRASAWA, H. (1968) A new silicate rock standard, JB-1 issued from the Geological Survey of Japan. Geochem. J. 2, 185. 
KuRASAWA, H. (1970) Private communication, Geological Survey of Japan, Kawasaki, Japan. KuRASAWA, H. (1974) Private communication, Geological Survey of Japan, Kawasaki, Japan. MAHON, M. (1974) Private communication, Department of Geophysics and Geochemistry, The Australian National University, Canberra, Australia.

MaruYama, G. and SudA, M. (1974) Private communication, Mitsubishi Mining Cement Research Laboratory, Yokose, Saitama, Japan.

MASUDA, Y. and YAGI, S. (1971) Private communication, University of Osaka Prefecture, Sakai, Japan.

MiYAKE, Y. and SUGIMURA, Y. (1967) Private communication, Tokyo Kyoiku University and Meteorological Research Institute, Tokyo, Japan.

MiYAKE, Y., SUgimURA, Y. and HIRAO, Y. (1970) Private communication, Tokyo Kyoiku University and Meteorological Research Institute, Tokyo, Japan.

MORI, T. (1972) Private communication, Kanazawa University, Kanazawa, Japan.

MUnSON, E. L. (1967) Private communication, U. S. Geological Survey, Denver, Colorado, U.S.A.

MURAD, E. (1971) Private communication, Mineralogisch-Petrographisches Institut der Universität, Tübingen, Germany.

MURAD, E. (1973) Determination of trace elements in unfused rock and mineral samples by X-ray fluorescence. Anal. Chim. Acta 67, 37-53.

NaGaSaWA, H. (1971) Private communication, NASA, Goddard, Maryland, U.S.A.

NAGURA, M. and IIDA, C. (1968) Atomic absorption spectrophotometric determination of cobalt, nickel, lead and copper in silicates with the absorption tube technique. Japan Analyst (Bunseki Kagaku) 17, 1513-1518 (in Japanese with English abstract).

NAKAMURA, N. and MASUdA, A. (1971) Private communication, Science University of Tokyo, Tokyo, Japan.

NishimURA, S. (1969) Radium and uranium content of standard rocks. Chem. Geol. 5, 139141.

-NISHIMURA, S. (1970) The determination of uranium content of standard rocks by fission track registration in muscovite. Radioisotopes 13, 194-196 (in Japanese with English abstract).

OAKLEY, P. J. (1973) Quoted in RANDALl, B. A. O. (1973).

OHTA, K. (1972) Private communication, Tokyo Coal and Mineral Laboratory, Tokyo, Japan.

Ozawa, T., Yoshida, M., Nagashima, S., IWASAKI, I. and ARIKaWa, Y. (1972) The sulfur content of igneous rocks in Japan. Annual Meeting Geochem. Soc. Japan, Abst. 13B10, 122 (in Japanese).

Ozima, M., Kaneoka, I., Kano, M., Kinoshita, K., OhnaKa, N., Nagata, T. and Karasa WA, H. (1968) Paleomagnetism and K-Ar ages of successive lava flow (2) - Kita-matsuura basalt, Kyushu, Japan. J. Geomag. Geoelect. 20, 85-92.

PRICE, V. (1969) Private communication, Furman University, Greenville, South Carolina, U.S.A.

RANDALL, B. A. O. (1972) Private communication, the University of Newcastle upon Tyne, Newcastle, England.

RANDLE, K. (1974) Some trace element data and their interpretation for several new reference samples obtained by neutron activation analysis. Chem. Geol. 13, 237-256.

RUBEŠKA, I. (1972) Private communication, Geological Survey of Czechoslovakia, Prague, Czechoslovakia.

RUBEŠKA, I. (1973) The determination of barium in gas shielded flames. Atom. Absorption Newsletter 12, No. 2, 33-36.

RUBEŠKA, I. (1974) Private communication, Geological Survey of Czechoslovakia, Prague, 
Czechoslovakia.

SAKURAMOTO, Y. (1973) Private communication, Osaka City University, Osaka, Japan.

SATO, N., KATO, T. and SUZUKI, N. (1974) Multielement photon activation analysis of rock materials with $30 \mathrm{MeV}$ bremsstrahlung. Radiochim. Acta 19, 1-6.

SATO, S., IIZUMI, S., and KaGAMI, H. (1969) Variance in measurement of Rb and Sr isotopic ratios of the sample, and its effect to the age determination (as to the standard sample JG-1). Mass Spectros. 17, 522-529.

SCHAFER, M. (1973) Private communication, Victoria University of Wellington, Wellington, New Zealand.

SCHMIDT, K. (1972) Private communication, Zentrales Geologisches Institut, Berlin, DDR.

SEKINE, S. and MOchIZUKI, T. (1968) Private communication, Geological Survey of Japan, Kawasaki, Japan.

SEN GUPTA, J. G. (1968) Private communication, Geological Survey of Canada, Ottawa, Canada.

SHIBATA, K. (1968) K-Ar age determinations on granitic and metamorphic rocks in Japan. Geol. Surv. Japan, Report No. 227, 1-71.

Shibata, K., NozAWA, T. and Wanless, R. K. (1970) Rb-Sr geochronology of the Hida metamorphic belt, Japan. Can. J. Earth Sci. 7, 1383-1401.

SHIBATA, K. and ADACHI, M. (1972) Rb-Sr and K-Ar geochronology of metamorphic rocks in the Kamiaso conglomerite, Central Japan. J. Geol. Soc. Japan 78, 265-271.

ShibatA, K. and UChIUMI, S. (1974) Private communication, Geological Survey of Japan, Kawasaki, Japan.

Shima, MaKoto (1968) Private communication, Institute of Physical and Chemical Research, Yamato-cho, Saitama, Japan.

Shirahise, T. (1974) Private communication, Geological Survey of Japan, Kawasaki, Japan.

ShIRAHATA, H. (1972) Private communication, Muroran Institute of Technology, Muroran, Japan.

SteElE, K. F. (1971) Private communication, University of of Arkansas, Fayetteville, Arkansas, U.S.A.

TAKeUCHI, S. and ShIma MASAKo (1968) Private communication, Institute for Solid State Physics, University of Tokyo, Tokyo, Japan.

TAmurA, S. (1968) Private communication, Japan Atomic Research Institute, Tokai, Ibaragi, Japan.

TANAKA, TAMOTSU and IIDA, C. (1969) Atomic absorption spectrophotometric determination of lead by absorption tube technique. Japan Analyst (Bunseki Kagaku) 18, 492-496 (in Japanese with English abstract).

TANAKA, Tsuyoshi and Masuda, A. (1971) Private communication, Geological Survey of Japan and Science University of Tokyo, Kawasaki and Tokyo, Japan.

TANAKA, TSUYOSHI (1974) Private communication, Geological Survey of Japan, Kawasaki, Japan.

TANIGUCHI, S. and OKADA, S. (1968) Private communication, Radiation Center of Osaka Prefecture, Sakai, Japan.

TERADA, K. (1974) Private communication, Kanazawa University, Kanazawa, Japan.

Terada, K., TERAShima, K. and KibA, T. (1972) Neutron activation analysis of selenium and osmium in rocks and sediments by direct evolution with the condensed phosphoric acid reagents. Annual Meeting, Geochem. Soc. Japan, Abst. 13Bll, 123 (in Japanese).

TERADA, K. and HiRAKaWA, S. (1974) Determination of chlorine in rocks by direct evolution with the condensed phosphoric acid - Chlorine contents of the standard rocks, JG-1 and JB-1 Annual Meeting, Geochem. Soc. Japan, Abst. C9, 131 (in Japanese). 
TERADA, K., OOBA, T. and KiBA, T. (1975) Separation and determination of selenium in rocks, marine sediments and plankton by direct evolution with the bromide-condensed phosphoric acid reagent. Talanta 22, 41-49.

Terashima, S. (1970) Private communication, Geological Survey of Japan, Kawasaki, Japan.

TERASHIMA,'S. (1971a) Atomic absorption analysis of minor elements in silicates. Japan Analyst (Bunseki Kagaku) 20, 321-326 (in Japanese with English abstract).

Terashima, S. (1971b) Private communication, Geological Survey of Japan, Kawasaki, Japan.

Terashima, S. (1973a) Atomic absorption analysis of beryllium and vanadium in rocks. Japan Analyst (Bunseki Kagaku) 22, 1317-1323 (in Japanese with English abstract).

Terashima, S. (1973b) Atomic absorption analysis of $\mathrm{Be}, \mathrm{V}, \mathrm{Ba}$ and $\mathrm{Rb}$ in rocks and flame emission analysis of Rb. Bull. Geol. Surv. Japan 24, 469-485 (in Japanese with English abstract).

TERASHIMA, S. (1974a) Spectrophotometric determination of chlorine and fluorine in the standard silicate rocks. Bull. Geol. Surv. Japan 25, 175-179.

TERASHIMA, S. (1974b) Atomic absorption analysis of micro-amounts of arsenic and antimony in silicates by the arsine and stibin generation method. Japan Analyst (Bunseki Kagaku) 23, 1331-1337 (in Japanese with English abstract).

Terashima, S. (1974c) Private communication, Geological Survey of Japan, Kawasaki, Japan.

ThompSON, G. (1972) Private communication, Woods Hole Oceanographic Institute, Woods Hole, Massachusetts, U.S.A.

TODA, N. (1973) Private communication, Nihon Cement Research Laboratory, Tokyo, Japan.

TOGASHI, Y. (1972) Private communication, Geological Survey of Japan, Tokyo, Japan.

Tomura, K., Higuchi, H., ONUmA, N. and Hamaguchi, H. (1968) Rapid determination of dysprosium in rock samples by neutron activation analysis with a $\mathrm{Ge}(\mathrm{Li})$ detector after chemical separation. Anal. Chim. Acta 42, 389-395.

TOMURA, K., Higuchi, H., TaKahashi, H., ONUMa, N. and Hamaguchi, H. (1968) Simultaneous determination of rubidium and cesium in rock samples by neutron activation analysis with a lithium-drafted germanium detector after chemical group separation. ibid. 43, 523-526.

UCHIDA, S. (1973) Private communication, Nihon cement Research Laboratory, Tokyo, Japan.

UEDA, N. (1968) Private communication, University of Tokyo, Tokyo, Japan.

UEDA, Y., AOKI, K., ONUKI, H. and KATO, Y. (1969) Analytical data on the geochemical standards JB-1 basalt and JG-1 granodiorite. J. Jap. Assoc. Min. Pet. Econ. Geol. 61, 35-39 (in Japanese with English abstract).

WEIGAND, P. W. (1970) Major and trace element geochemistry of the Mesozoic dolerite dikes from eastern North America, Ph. D. Thesis, University of North Carolina, Chapel Hill, North Carolina, U.S.A.

YAMADA, J., IIDA, C. and YAMASAKI, K. (1969) Atomic absorption spectrophotometric determination of trace amount of cadmium in silicates with the absorption tube technique. Japan Analyst (Bunseki Kagaku) 18, 1088-1092 (in Japanese with English abstract).

YAMADA, J., IIDA, C. and YAMASAKI, K. (1970) Atomic absorption determination of cadmium in silicates with the absorption tube technique combined with a ring burner. ibid. 19, 12591263 (in Japanese with English abstract).

YamaGUCHI, M. (1968) Private communication, Kyushu University, Fukuoka, Japan. 